SHORT REPORT

\title{
Myotonia and flaccid dysarthria in patients with adult onset myotonic dystrophy
}

\author{
B J M de Swart, B G M van Engelen, J P B M van de Kerkhof, B A M Maassen
}

J Neurol Neurosurg Psychiatry 2004;75:1480-1482. doi: 10.1136/jnnp.2003.032151

Background: Myotonia and weakness are the most important components of dysarthric speech in myotonic dystrophy. Objective: To specify and quantify possible defects in speech execution in patients with adult onset myotonic dystrophy. Methods: Studies on speech production were done on 30 mildly affected patients with myotonic dystrophy. Special attention was paid to myotonia. Because muscle activity can result in a decrease of myotonia, speech characteristics were measured before and after warm up. The possibility that warming up causes increased weakness was also assessed. Results: As with other motor skills, a warm up effect was found in speech production, resulting in an increase in repetition rate and a decrease in variability of repetition rate. Signs of fatigue did not occur.

Conclusions: Warming up is valuable for patients with myotonic dystrophy in reducing the influence of myotonia on speech production.

M yotonic dystrophy (Steinert's disease) is an autosomal dominantly inherited disease characterised by progressive muscle weakness and myotonia. Although classified as a muscle disease, myotonic dystrophy is a multisystem disorder with a varying degree of internal, cardiac, and ophthalmic pathology. ${ }^{1}$ Myotonic dystrophy can be divided into three types based on the time of onset, the clinical features, and the number of CTG repeats in DNA. ${ }^{2-4}$ The disease is more severe and more progressive when maternally inherited, when the DNA repeats have greater length, and when it is of congenital or early onset. Congenital and juvenile onset myotonic dystrophy ( 1 to 12 years) is characterised by mental retardation, muscle weakness, multisystem pathology, rapid progression, and oral and maxillofacial manifestations. ${ }^{15-8}$ In adult onset myotonic dystrophy (12 to 50 years), muscle signs and myotonia predominate. Adult onset myotonic dystrophy also involves mental slowness but is mildly progressive and the multisystem pathology is variable. Late onset myotonic dystrophy (over 50 years) has few physical signs-mainly cataract, myotonia, and frontal balding.

As both weakness and myotonia are responsible for problems of speech production in patients with myotonic dystrophy, we investigated these two features in the present study. The hallmark of myotonia is a slowing down of relaxation of the muscles after rest or activity. Repeated muscle contractions can reduce myotonia, a feature known as the "warm up" phenomenon." The most reliable site for detecting myotonia is the hand.

Dystrophy and muscle weakness are prominent in the facial, jaw, and neck musculature, causing flaccid dysarthria. ${ }^{10-12}$ The quality of speech in patients with myotonic dystrophy depends on the severity of the condition; in general, hypotonia of the speech musculature results in monotony, hypernasality, hoarseness, shorter stretches of speech, a slow speech rate, and a decrease in volume and intelligibility. ${ }^{13-15}$

In addition to the effects of muscle weakness, the myotonia itself also affects speech production. Myotonia can cause irregularities in speech fluency, though its effects are unpredictable. Besides fluctuation in time, microanalysis of articulation suggests that the manner and place of articulation are related to the presence of myotonia. Some patients have more problems with bilabial sounds, others with alveolar or velar sounds. Plosive sounds cause more difficulty than fricatives, while talking loudly and shouting seem to provoke myotonia. ${ }^{15}$

Our first aim in this study was to determine the onset of myotonia in the speech musculature. Does myotonia disappear after warming up or is it possible that myotonia occurs or even increases during speech production? Our second aim was to determine whether flaccid dysarthria might increase after intensive speech activity as an adverse effect of warm up resulting from fatigue.

\section{METHODS}

\section{Subjects}

Adult outpatients with myotonic dystrophy were asked to participate in the study. There were three inclusion criteria: age at onset of first signs of myotonic dystrophy between 12 and 50 years; no cognitive impairment; and diagnosis confirmed by DNA linkage. ${ }^{36}$ Sixteen men and 14 women were included. Ages ranged from 18 years to 68 years (mean (SD), 40.4 (12.6) years). The duration of the disease ranged from 1 to 31 years ( 12.3 (8.2) years).

\section{Procedure}

A protocol was devised to collect information about fatigue, warm up, myotonia, and dysarthria. To demonstrate fatigue and warming up effects, we designed a protocol which elicited at least 10 minutes of continuous speech. To give patients the opportunity to present their best performance, they were asked to undertake all the tasks three times. The first and last task of the protocol-the maximum repetition rate (MRR) tasks-were identical. ${ }^{17-19}$ The purpose of duplication was to allow speech samples from the beginning of the examination to be compared with those at the end. In both series of MRR tasks, patients were asked to produce monosyllabic sequences [papapa..], [tatata..], and [kakaka..] and trisyllabic sequences [pataka..] as fast as possible.

Between these two series of MRR tasks, the subjects read aloud three minutes of text three times. On the first occasion, they were asked to use their habitual speech rate; on the second and third occasions they were asked to read the text as fast as possible. This variation stimulated patients to use their articulatory muscles intensively, and contributed to

Abbreviations: MRR, maximum repetition rate; MSP, maximum sound prolongation 
eliciting signs of fatigue. This task was followed by the maximum sound prolongation tasks (MSP tasks). Patients had to phonate the vowel "ah" [a:] and perform the voiceless consonants "s" [s:] and " $\mathrm{f}$ " [f:] for as long as possible. The last but one task was to recite the months of the year, then again a second and third time again as fast as possible.

With a Marantz CP 230 cassette recorder and a Sony ECM stereo microphone, all speech tasks were recorded on audiotape (TDK high position SA 60).

\section{Acoustic analysis}

The three productions of all MRR and MSP tasks were digitised at a sampling frequency of $10 \mathrm{kHz}$ by a DASH AD/ DA converter (Kay 4300B). The fastest MRR tasks and the longest MSP tasks of each task were selected for analysis. For all MRR tasks the repetition rate, intensity, and variability in speech rate were measured. For this, tags were inserted in the acoustic signal by hand for each syllable at burst onsets. The last syllable in each series was excluded from analysis. On the basis of the tags, syllable durations were measured and transferred to SPSS 10.0. For the MSP tasks only the duration of the acoustic signal was measured.

The speech rate for the reading task was calculated by counting the number of syllables.

\section{Statistical analyses}

All data were analysed with SPSS 10.0. Correlations between tasks were calculated using Pearson's correlation coefficient. The difference between the values at the beginning and at the end of the examination were determined using univariate analysis of variance tests with repeated measures design (within-subject factors are pre/post measurements and MRR tasks). Comparisons of results of our measurements with the values of studies in healthy subjects were done with onesample $t$ tests.

\section{RESULTS}

In the MRR measurements (fig 1), especially at the beginning, significantly fewer [ka] syllables were produced per second than [pa] and [ta] syllables. Compared with healthy controls only the repetition rate of [ka] at the beginning of the examination was significantly lower $(t=-2.487 ; \mathrm{p}=0.019) .{ }^{20}$ Trisyllabic sequences were produced faster $(t=4.381 ; \mathrm{p}=0.000)$ than monosyllabic sequences, as has been found in healthy speakers. ${ }^{21}$ Comparing the MRR productions using an analysis of variance test, a significant pre/post effect was found $\left(F_{(1,28)}=14.478, p<0.001\right)$. Figure 1 shows faster performances for all four MRR sequences, but suggests a smaller pre/post effect for [pa] than for [ta], [ka], and [pataka] sequences. This was confirmed by a significant effect for the interaction between the factors pre/post * $\operatorname{task}(\mathrm{F}=3.026, \mathrm{df}$ $=3, \mathrm{p}=0.034$ ).

The mean intensity in all MRR tasks decreased at the end of the protocol, but none of the changes in intensity was significant (fig 2).

In all patients the variability of the repetition rate of monosyllabic sequences in the first measurement was greater than the variability in the second measurement $(t=8.182$; $\mathrm{p}=0.000)$.

The mean speech rate of patients reading the first 100 syllables of the text was not found to be significantly different from the rate at which they read the last 100 syllables.

In the MSP task the mean prolongation duration of [a:], [s:], and [f:] (14.56, 11.35, and 7.21 seconds) was significantly less than prolongation times of healthy controls in a former study by Maassen et $\mathrm{al}^{15}$ ([a:], $t=-8.012, \mathrm{p}=0.000$; [s:], $t=-19.053, \mathrm{p}=0.000 ;[\mathrm{f}:], t=-13.661, \mathrm{p}=0.000)$.

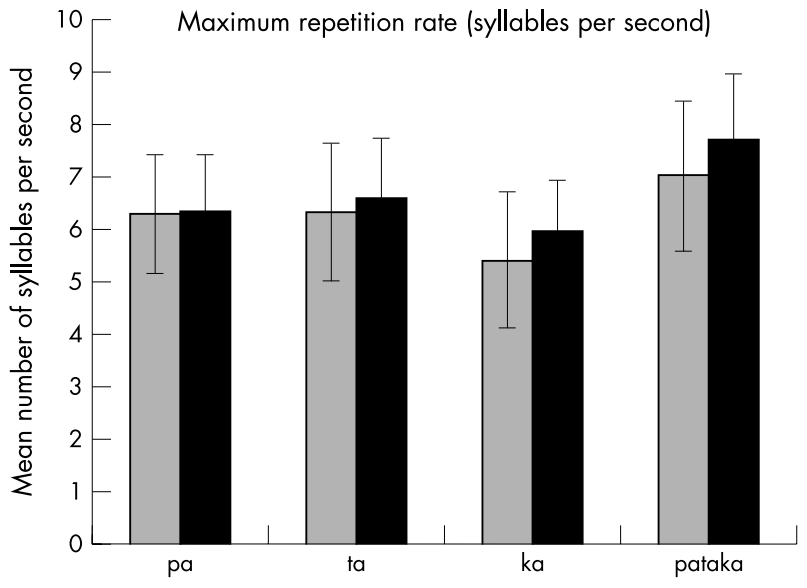

Figure 1 Number of syllables per second for the monosyllabic sequences [pa], [ta], and [ka], and the trisyllabic sequence [pataka]. The grey bars, representing the values at the beginning of the examination, are shorter than the black bars, which represent the values at the end of the examination, when the muscles have "warmed up".

High correlations were found between performances of separate speech tasks, establishing the item to item reliability. All correlations between tasks concerning repetition rate $(r=0.65$ to $r=0.95 ; \mathrm{p} \leqslant 0.01)$ and sound prolongation $(r=0.58$ to $r=0.96 ; \mathrm{p} \leqslant 0.01)$ were significant.

\section{DISCUSSION}

We found that the occurrence of myotonia in the speech musculature was significantly more prominent when patients with myotonic dystrophy started speaking. The influence of myotonia decreased during the production of continuous speech as a result of warming up, causing a significant increase in the number of syllables per second that could be produced and a decrease in variability. The repetition rate at the end of the examination was near the values of healthy subjects for all MRR tasks $(t=12.677 ; \mathrm{p}<0.001) .^{20}$

Especially at the start, patients with myotonic dystrophy produced significantly fewer [ka] syllables than [pa] or [ta]. This phenomenon is not seen to the same extent in healthy subjects. ${ }^{20}$ A possible explanation could be that more muscle activity or tension is required for [ka], with the result that myotonia has a greater effect. After warming up, the greatest increase in repetition rate was seen in the two tasks containing the syllable [ka]-that is, [kakaka..] and [pataka..]. In contrast, the repetition rate of the syllable

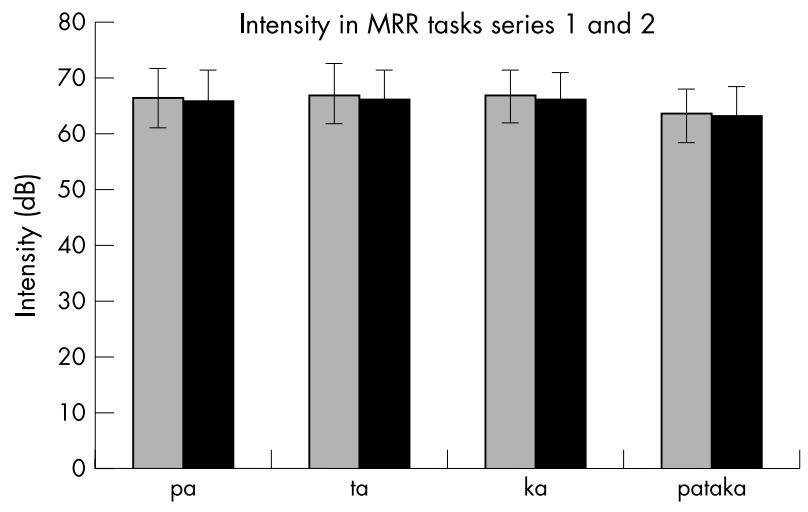

Figure 2 Intensity in $\mathrm{dB}$ for the monosyllabic sequences [pa], [ta], and [ka] and the trisyllabic sequence [pataka]. The grey bars represent the intensity at the beginning of the examination and the black bars at the end of the examination when the muscles have "warmed up". 
[pa] did not show a significant increase. During MRR tasks, the activity and muscle tension in lip and jaw muscles to produce the syllable [pa] seem to be too small to provoke a major myotonic disturbance.

Despite the decrease in variability during speech production, in clinical practice some patients report an increase in problems during conversation. Two possible explanations for the occurrence of problems in daily conversation are, first, that a patient's speech production in some daily conversations may be too short to cause a warm up effect; and second, that pauses between speaking may be too long to allow the effect of warming up to be maintained.

Signs of flaccid dysarthria are shown in maximum sound prolongation tasks, where respiratory-phonatory weakness caused by the dystrophy leads to impaired performance. Our investigation of intensity as an indicator for the occurrence of fatigue showed no significant decrease in intensity of MRR tasks after periods of continuous speaking. The fact that the mean speech rate while reading the first 100 syllables was not significantly different from the speech rate for the last 100 syllables indicates that warming up was already effective at this point in the protocol, and persisted during further tasks. There was no evidence that fatigue occurred.

\section{Conclusions}

Compared with healthy subjects, patients with adult onset myotonic dystrophy show signs of myotonia and weakness as components of flaccid dysarthria (lower repetition rate, reduced loudness, limited duration of sound prolongation, and greater variability). Patients also show a warm up effect in speech production. This results in an increase in repetition rate and a decrease in myotonia and variability, while signs of fatigue-such as an adverse effect of long and intensive use of the speech musculature-do not occur. Thus warming up is valuable in reducing the influence of myotonia on speech production.

\section{Authors' affiliations}

B J M de Swart, B G M van Engelen, Neuromuscular Centre and Institute of Neurology, University Medical Centre Nijmegen, Nijmegen, Netherlands

J P B M van de Kerkhof, Department of Speech and Language, University of Niimegen

B A M Maassen, Medical Psychology and Child Neurology Centre, University Medical Centre Nijmegen

Competing interests: none declared

Correspondence to: B J M de Swart, Neuromuscular Centre Nijmegen, Institute of Neurology, University Medical Centre Nijmegen, PO Box 9101, 6500 HB Nijmegen, Netherlands; b.deswart@neuro.umcn.nl
Received 10 November 2003

In revised form 19 December 2003

Accepted 21 December 2003

\section{REFERENCES}

1 Harper PS. Myotonic dystrophy. Philadelphia: WB Saunders Co, 1989:1192-215.

2 Koch M, Grimm T, Harley H, et al. Genetic risks for children of women with myotonic dystrophy. Am J Hum Genet 1991;48:451-70.

3 Brunner HG. Genetic studies in myotonic dystrophy. Nijmegen: University of Nijmegen, 1993. [Doctoral dissertation.]

4 ter Bruggen JP. Myotonic dystrophy: a quantification of some clinical aspects of the classical form. Nijmegen: University of Nijmegen, Department of Medical Sciences, 1994.

5 Huber S, Kissel J, Shuttleworth E, et al. Magnetic resonance imaging and clinical correlates of intellectual impairment in myotonic dystrophy. Arch Neurol 1989;46:536-40.

6 Hashimoto T, Tayama M, Miyazaki M, et al. Neuroimaging study of myotonic dystrophy. I. Magnetic resonance imaging of the brain. Brain Dev 1995; 17:24-7

7 Peñarrocha M, Bagan JV, Vilchez J, et al. Oral alterations in Steinert's myotonic dystrophy: a presentation of two cases. Oral Surg Oral Med Oral Pathol 1990;69:698-700

8 Mercier J, Bennani F, Ferri J, et al. Maxillofacial manifestations of Steinert's myotonic dystrophy. Clinical and therapeutic aspects. Rev Stomatol Chir Maxillofac 1995:96:74-82.

9 Cooper RG, Stokes MJ, Edwards RH. Physiological characterisation of the "warm-up" effect of activity in patients with myotonic dystrophy. J Neurol Neurosurg Psychiatry 1988;51:1134-41.

10 Ramig LA, Scherer RC, Titze IR, et al. Acoustic analysis of voices of patients with neurologic disease: rationale and preliminary data. Ann Otol Rhinol Laryngol 1988;97:164-72

11 Salomonson J, Kawamoto H, Wilson J. Velopharyngeal incompetence as the presenting symptom of myotonic dystrophy. Cleft Palate J 1988;25:296-300.

12 Weinberg B, Bosma JF, Shanks JC, et al. Myotonic dystrophy initially manifested by speech disability. J Speech Hear Disord 1968;33:51-9.

13 Darley FL, Aronson AE, Brown JR. Motor speech disorders. Philadelphia: WB Saunders Co, 1975

14 McNeil MR. Clinical management of sensorimotor speech disorders. Stuttgart: Thieme, 1997.

15 Maassen B, ter Bruggen JP, Nanninga-Korver A, et al. Speech motor programming and execution in myotonic dystrophy: a quantification of some clinical aspects of the classical form. J Med Speech Lang Pathol 1995;3:85-93.

16 Brunner H, Coerwinkel-Driessen M, Smeets B, et al. Definition of subchromosomal intervals around the myotonic dystrophy locus at 19q. In: Bartsocas B, ed. Genetics of neuromuscular disorders. New York: AR Liss, 1989.

17 Thoonen G, Maassen B, Gabreels FJM, et al. The integrated use of maximum performance tasks in differential diagnostic evaluations among children with motor speech disorders. Clin Linguistics Phonetics 1996;10:311-36.

18 Wit J, Maassen B, Gabreëls F, et al. Traumatic versus perinatally acquired dysarthria: assessment by means of speech-like maximum performance tasks. Dev Med Child Neurol 1994;36:221-9.

19 Kent RD, Kent JF, Rosenbek JC. Maximum performance tests of speech production. J Speech Hear Disord 1987;52:367-87.

20 Kent RD. Reference manual for communicative sciences and disorders; speech and language. Texas: Pro-ed Inc, 1994.

21 Wit J, Maassen B, Gabreëls FJM, et al. Maximum performance tests in children with developmental spastic dysarthria. J Speech Hear Res 1993;36:452-9. 